\title{
https://doi.org/10.46344/JBINO.2020.v09i05.18
}

\section{A REVIEW ON TYPE OF INFECTIONS IN PATIENTS UNDERGOING HEMODIALYSIS}

\author{
Divya Joy, L.Panayappan*, K.Krishnakumar
}

\begin{abstract}
, Department of Pharmacy Practice, St. James' college of Pharmaceutical sciences and St. James' Hospital Trust Pharmaceutical Research Centre (DSIR Recognized), Chalakudy, Kerala
\end{abstract}

(Received on Date: $18^{\text {th }}$ May 2020

Date of Revision\& Acceptance: $24^{\text {th July }} 2020$

Date of Publish: 1st September 2020)

\section{Email: panayappan76@gmail.com, stjamespharmacyproject@gmail.com}

\begin{abstract}
End Stage Renal Disease (ESRD) is a medical condition which is resulted when kidneys cease functioning on permanent basis which requires dialysis or transplantation for maintenance of life. Infections are most common cause of mortality and morbidity in ESRD patients. Dialysis have become more prominent nowadays due to significant rise in kidney failure. Infections in ESRD patients are mainly due to immune suppression, comorbid conditions, malnutrition and older age. The infections that affects the dialysis patients include fistula or graft related infection, catheter related infections, Hepatitis B, Hepatitis C, Vancomycin resistant enterococci, Methicillin resistant staphylococcus aureus, tuberculosis, HIV. This article reviews regarding the type of infections in ESRD patients and provide an in-depth understanding. ESRD patients must be routinely checked for preventing occurrence of infections. Expeditious diagnosis and appropriate therapy may reduce the risk of infections.
\end{abstract}

Key-words: Risk Of Infection, Pathogen ,Infected Patient, Immunodeficiency

\section{No: of References: 22}




\section{INTRODUCTION}

Infection is the common cause of morbidity and mortality in end stage renal disease (ESRD) patients'. Infections are recurrent cause of hospitalization and is the second leading cause of mortality followed by cardiovascular disease in ESRD patients 2 . End stage Renal Disease (ESRD) is a medical condition which develops when a person's kidneys cease functioning on a permanent basis leading to the need for a regular course of long term dialysis or a kidney transplantation to maintain life .ESRD patients are more vulnerable to the infection since they are immunocompromised, have co-morbid conditions such as diabetes, malnutrition and may be of advanced age ${ }^{1}$.

Infection in ESRD patients may be of two types which is access related or nonaccess related. Access related infection include infection through the fistula or graft and catheter. Non access related infection in dialysis patients include hepatitis, respiratory tract infection, tuberculosis, HIV, urinary tract infection.

\section{Fistula or graft related infection}

An arteriovenous (AV) fistula has the lowest infection rate $^{4}$. The known risk factors for infection through AV fistula can be through IV drug use, dermatitis or scratching over fistulas and grafts, and poor hygiene 5,6 . Invasive procedures such as angioplasty, thrombectomy, or graft revision also increase the risk of infection' ${ }^{6}$. Hemodialysis patients have thrice the colonization rate than chronic renal failure (CRF)patients not on hemodialysis 1 . Infection is suspected if AV access shows erythema, warmth, tenderness, induration, or purulent drainage.

\section{Catheter related infection}

ESRD patients without fistula or graft access or ESRD patients undergoing dialysis require catheterl. S.aureus and S.epidermis are most common pathogens responsible for catheter related infection' ${ }^{1}$.Catheter related infection rate is higher than a fistula or graft. Prolonged catheterization, frequent manipulation, improper aseptic techniques are the risk factors of infection [15-17]. Different type of catheter related infections includes exit site infection, tunnel infection, catheter -related septicemia, septic thrombophlebitis, and infusate related bacteremia/ fungemia. Catheter exit site infection constitutes inflammation at the catheter exit site without blood infection. Redness and tenderness along the catheter's subcutaneous track indicate tunnel infection. Catheter related septicemia requires exit site or tunnel infection, the same organism in blood cultures and no other source of septicemia. An infected thrombus in the catheter or vessel defines septic thrombophlebitis. Infusion of contaminated fluid may arise infusate related septicemia.

\section{Hepatitis B}

Hemodialysis patients may acquire HBV infection from community sources, or from 
transmission in hemodialysis centers due to inadequate infection control precautions or to accidental breaks in technique?.

Transmission: HBV is transmitted by percutaneous or permucosal route by exposure to infectious blood or to body fluids that contain blood and through chronically infected person. HBsAg can be detected in dialysis centers on clamps, scissors, dialysis machine knobs and doorknobs [18]. So, blood -contaminated surfaces which are not cleaned and disinfected are the reservoirs for HBV transmission. Dialysis staff members can transfer the virus from contaminated surface to the patients by their hands or gloves or through the use of contaminated equipments.HBV infection can be spread through the multiple dose medication vials and intravenous solution which were used by multiple personnel's, medications for injection that were prepared at the adjacent areas where blood samples are handled[19,20].

\section{Prevention:}

The transmission of HBV infections can be prevented by adopting suitable measures such as serologic surveillance of patients and staff through monthly testing of susceptible patients for $\mathrm{HBsAg}$, isolation of HBsAg positive patients, assignment of staff members to HBsAg -positive patients and not to HBV susceptible patients during the same shift,assignment of dialysis equipment to separate to the HBsAg positive patients and HBV susceptible patients,cleaning and disinfection of nondisposable items before its use on another patient,usage of gloves while touching on equipment or any patient and replace with new one while touching other patient[21].

Risk Factors: It includes treatment of an acute or chronic HBV previously infected patient, accepting HBsAg positive patients and not treating these patients with a separate room and machine; presence of $<50 \%$ patients vaccinated against HBV. HBsAg positive patients are a prospective source of nosocomial transmission.

Clinical manifestations: HBV can be acute or chronic hepatitis. It includes 45-160 days of incubation period. Infants, young children and immunosuppressed adults with newly acquired HBV infection are usually asymptomatic. HBV infections associated with anorexia, malaise, nausea, vomiting, abdominal pain and jaundice.

Treatment: Chronic hepatitis B patients are treated by monotherapy with alpha interferon or lamivudine. Dosage adjustment must be done in case of renal impairment patients.

\section{Hepatitis C}

Hepatitis $C$ is a disease principally transmitted by the blood borne route in hemodialysis patients. About $75 \%$ of cases of Hepatitis $C$ in adults are asymptomatic [3].

Transmission: $\mathrm{HCV}$ is transmitted by percutaneous exposure to infectious blood[22] It is also transmitted through 
equipment and supplies that were not disinfected between patient use, use of common medication carts to prepare the medications, sharing of multiple dose medication vials which were placed on the top of hemodialysis machines, contaminated priming buckets which were not cleaned and disinfected, blood spills that were not cleaned[21].

Clinical manifestations: HCV causes acute and chronic hepatitis. The incubation period ranges from 14-180 days. Newly acquired patients are asymptomatic or have a mild clinical illness. Hemodialysis patients with newly acquired HCV characterized by elevated serum ALT levels.

Treatment: Alpha interferone alone or in combination with ribavirin.

\section{Vancomycin -Resistant Enterococci (VRE)}

Vancomycin resistant enterococci was first reported in 1989 among renal failure patients in England has emerged as a major nosocomial pathogen. The percentage of enterococcal isolates from all body sites that were resistant to vancomycin increased from 0.3\% in 1989 to $10.5 \%$ in 1997 [3]. Risk factors for developing VRE infection include severity of illness and receipt of antibiotics particularly vancomycin [3].

\section{Methicillin-Resistant Aureus (MRSA)}

Staphylococcus

S.aureus is the common cause of vascular access infection in hemodialysis patients
[11,12]. MRSA denotes that the Staphylococcus aureus resistant to the methicillin antibiotics.There was an increase in the rate of methicillin resistant strain from 1975 to 1991 [8].

\section{Tuberculosis}

ESRD patients who have tuberculosis without active disease may develop into active tuberculosis due to ESRD associated immunodeficiency. CDC recommends that ESRD patients must be screened by tuberculin test for tuberculosis and must be given preventive therapy if infected [13,14]. CDC also recommends that medical care facilities should assess the risk of nosocomial transmission of Mycobacterium tuberculosis and develop an appropriate plan according to level of risk.

\section{Human Immunodeficiency Virus (HIV)} Infection

Transmission of HIV infection in dialysis has been reported in developing countries.HIV infection transmitted from known HIV infected patients to staff member through needlestick injury [9,10]. Patients with HIV infection can receive the dialysis without additional precautions like isolation from other patients by either room or machine.

Diagnosis: Assays that measure antibody to HIV and continuous EIA positive test which is confirmed by Western blot are usually done to diagnose the HIV infection.

\section{Conclusion}


ESRD patients are infected during the dialysis process. The infection occurs since they are immune compromised due to decline in the health status with respect to chronic kidney. Infection can be recognized by identification of signs and symptoms, medication usage, laboratory investigations. Initial recognition of infection may prevent hastening to the complications. The rate of type of infections developed may assimilate according to the patient's condition, dialysis procedures.

\section{References}

Susie Q.Lew and Kianoosh Kaveh: Dialysis access related infections, ASAIO Journal 2000;46:S6-S12.

Shu-Hong Bi, Christopher R Blagg, Suhail Ahmad, et al. Infection Related Hospitalizations in Home Hemodialysis Patients.Blood Purif 2015:40:187-193.

Jerome I.Tokars, Miram J Alter, Matthew J.Arduino: National Surveillence Of DialysisAssociated Disease In The United States, 1997.

Kherlakian GM, Roedersheimer LR, Arbaugh JJ, Newmark KJ, King LR: Comparison of autogenous fistula versus expended polytetrafluoroethylene graft fistula for angioaccess in hemodialysis. Am J Surg 152:238-243,1986.

Keane WF,Shapiro FL, Raji L: Incidence and Type of Infections occurring in 445 chronic hemodialysis patients.Trans ASAIO 23:41$46,1997$.
Kaplowitz LG, Comstock JA, Landwehr DM, Dalton HP, Mayhall CG: A prospective study on infections in hemodialysis patients: Patient hygiene and other risk factors for infection.Infect Control Hosp Epidemiol 9:534-541,1988.

Alter MJ, Ahtone J,Maynard JE: Hepatitis B virus transmission associated with a multiple dose vial in a hemodialysis unit.Ann Intern Med. 1983;99:330-3

\section{Panlilio AL ,Culver DH,Gaynes} RP:Methicillin-resistant Staphylococcus aureus in U.S hospitals, 1975-1991. Infect Control Hosp Epidemiol,1992;13:582-6.

Hassan NF,El Ghorab NM,Abdel Rehim MS :HIV infection in renal patients in Egypt.Acquired Immune Deficiency Syndrome.1994;8:853

Velandia M Fridkin SK,Cardenas V: Transmission of HIV in dialysis centre.Lancet.1995;345:1417-22.

Kaplowitz LG,Comstock JA, Landwehr DM,Dalton HP ,Mayhall CG.A prospective study of infections in hemodialysis patients:patient hygiene and other risk factors for infection.Infect Control Hosp Epidemiol.1988;534-41

Kessler M, Hoen B,Mayeux D, Hestin D,Fontenaille $C$.Bacteremia in patients on chronic hemodialysis.Nephron 1993;64:95100.

Centers for Disease Control and Prevention. Screening for tuberculosis and 
tuberculous infection in high -risk populations.MMWR.1995;44:1-34

Tokars JI, Miller B .tuberculin skin testing of esrd patients.Am J Kidney Dis.1997;30;4567.

\section{Marr KA,Sexton D,Conlon P,Corey} R,Schwab SJ,Kirkland K:Catheter -related bacteremia and outcome of attempted catheter salvage in patients undergoing hemodialysis.Ann Intern Med 127:275$280,1997$.

Suhocki PV, Conlon PJ, Knelson MH, Harland R, Schwab SJ: Silastic cuffed catheters for hemodialysis vascular access: Thrombolytic and mechanical correction of malfunction. Am J Kidney Dis.1996; 28: 379-386.

Jaar B, Furth SL, Hermann J,et al. Septicemia patients:Incidence,risk factors and prognosis. Kidney Int 55:1081-1090,1999.

Favero MS, Maynard JE, Petersen NJ, et al. Hepatitis-B antigen on environmental surfaces [Letter]. Lancet 1973;2:1455.

CDC. Vaccinations for adults with chronic kidney failure and hemodialysis programCalifornia, Nebraska, and Texas, 1994. MMWR 1996;45:285-9

\section{Snydman DR, Bryan JA, Macon EJ, Gregg}

MB: Hemodialysis-associated hepatitis: report of an epidemic with further evidence on mechanisms of transmission. Am J Epidemiol 1976; 104:563-70.
Centers for Disease Control and Prevention. Recommendations for preventing transmission of infections among chronic hemodialysis patients. MMWR 2001;50(No. RR-5).

Moyer LA, Alter MJ. Hepatitis C virus in the hemodialysis setting: a review with recommendations for control. Semin Dial 1994;7:124-7.

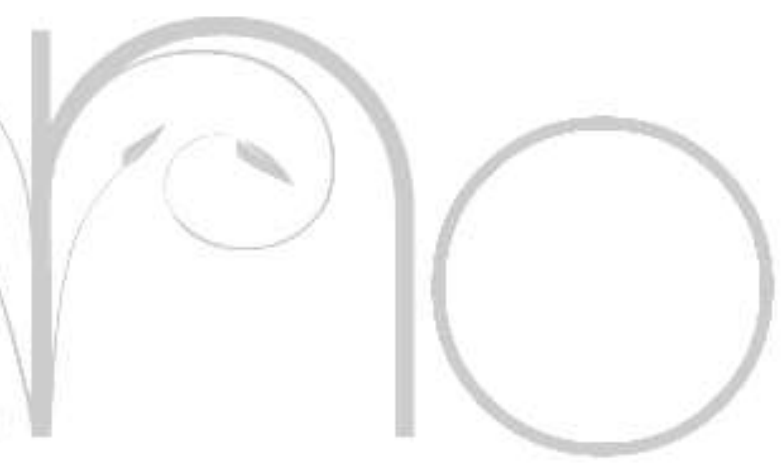

